BY CLAUDIO DELRIEUX, VIRGINIA BALLARÍN, CRISTIAN GARCIIA BAUZA, AND MARIO A. LÓPEZ
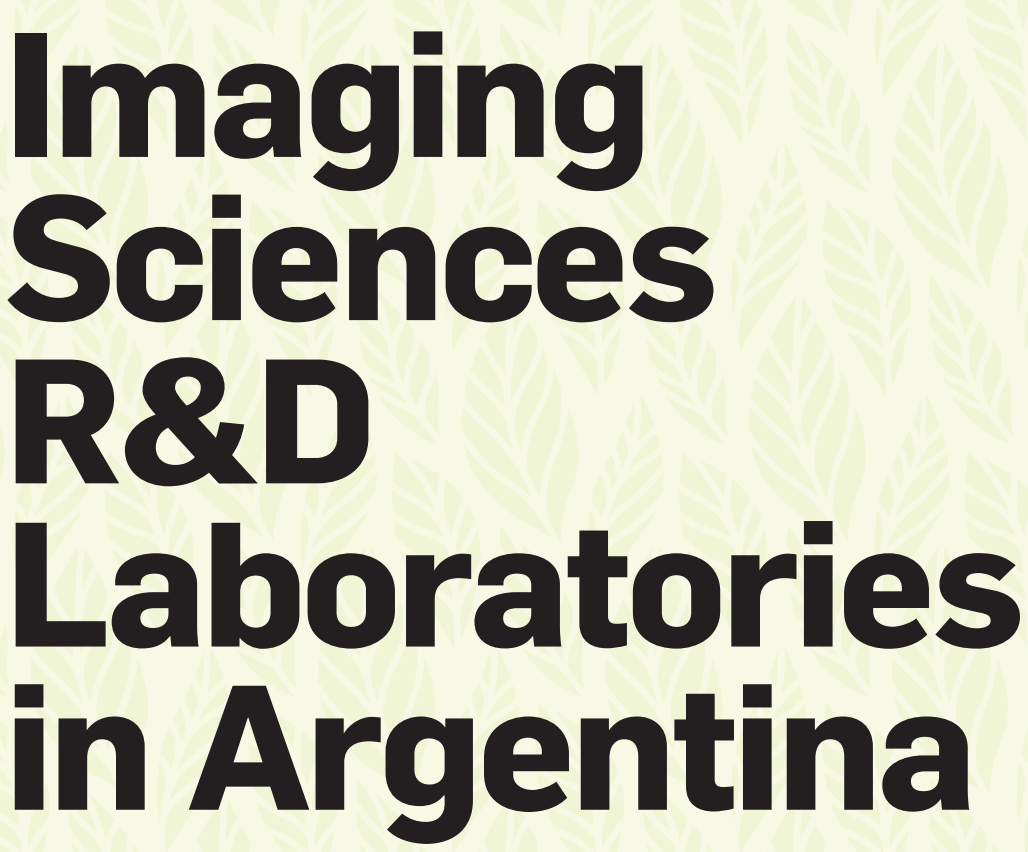

WE USE THE term imaging sciences to refer to the overarching spectrum of scientific and technological contexts which involve images in digital format including, among others, image and video processing, scientific visualization, computer graphics, animations in games and simulators, remote sensing imagery, and also the wide set of associated application areas that have become ubiquitous during the last decade in science, art, human-computer interaction, entertainment, social networks, and many others. As an area that combines mathematics, engineering, and computer science, this discipline arose in a few universities in Argentina mostly in the form of elective classes and small research projects in electrical engineering or computer science departments. Only in the mid-2000s did some initiatives aiming to generate joint activities and to provide identity and visibility to the discipline start to appear. In this short paper, we present a brief history of the three laboratories with the most relevant research and development (R\&D) activities in the discipline in Argentina, namely the Imaging Sciences Laboratory of the Universidad Nacional del Sur, the PLADEMA Institute at the Universidad Nacional del Centro de la Provincia de Buenos Aires, and the Image Processing Laboratory at the Universidad Nacional de Mar del Plata.

The Imaging Sciences Laboratory ${ }^{a}$ of the Electrical and Computer Engineering Department of the Universidad Nacional del Sur Bahía Blanca began its activities in the 1990s as a pioneer in Argentina and Latin America in research and teaching in computer graphics, and in visualization. The facility currently is staffed by six National Research and Technology Council (CONICET) fellows (Claudio Delrieux, Alejandro Vitale, Felix Thomsen, Natalia Revollo, Marina Cipolletti, and Noelia Revollo), plus three postdocs and 13 Ph.D. candidates, who are actively researching novel image analysis methods including multifractality, complexity theory, and deep learning in the hope of generating breakthroughs in research contexts including three-dimensional (3D) medical images, ${ }^{1}$ 3D shape analysis, ${ }^{2}$ biometrics, ${ }^{3}$ biomedical signal analysis, ${ }^{4}$ microscopy,${ }^{5}$ remote sensing: ${ }^{6}$ satellite imagery, ${ }^{7}$ and environmental monitoring, ${ }^{8}$ while maintaining research and development activities in scientific visualization and computer graphics.

In Figure 1, we show novel 3D texture analysis techniques applied to brain MRIs in mild and moderate Alzheimer's disease patients (and in comparison with a control population of subjects of similar age and condition). The proposed operators detect compromised areas of the subjects' brains that correlate to their actual

a www.imaglabs.org 
Figure 1. Per-voxel statistical significance of different novel three-dimensional texture analyses and their correlation to different degrees of Alzheimer's disease. The operators detect compromised areas in the subjects' brains that correlate to their actual neurocognitive impairments. ${ }^{1}$

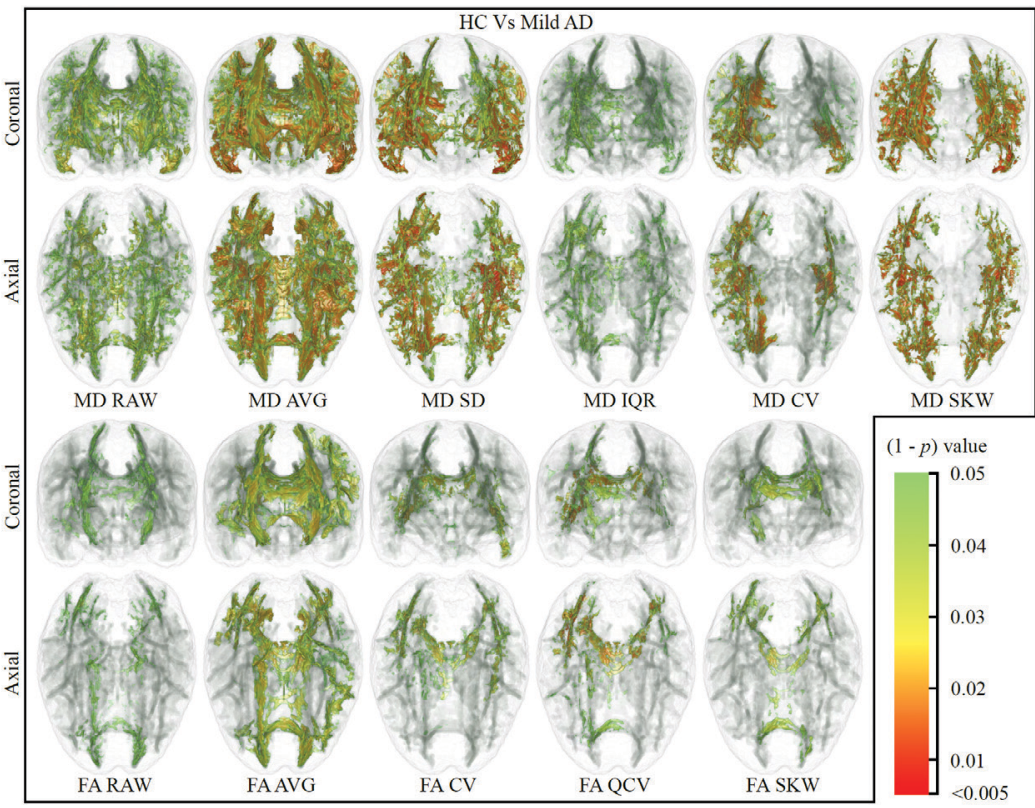

Figure 2 (Left): A point cloud generated with handheld devices using photogrammetry and deep learning. (Right): A 250-person body shape and overweight analysis shows that BMI and similar indices may be misleading, and proposes different assessment strategies. ${ }^{3}$
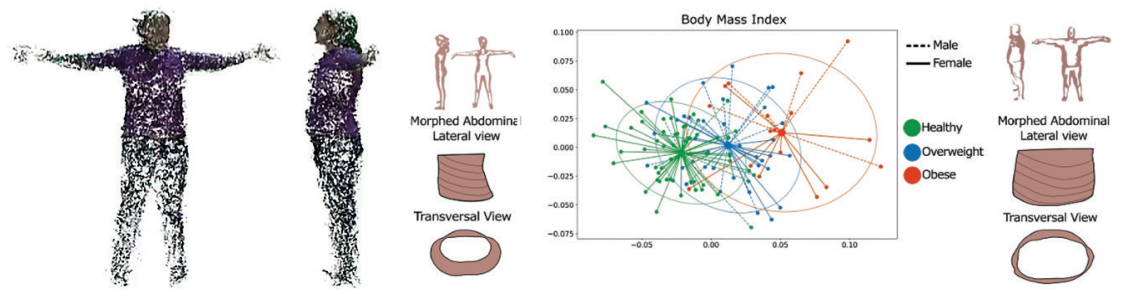

Figure 3. Subway train simulators for the Buenos Aires city network.

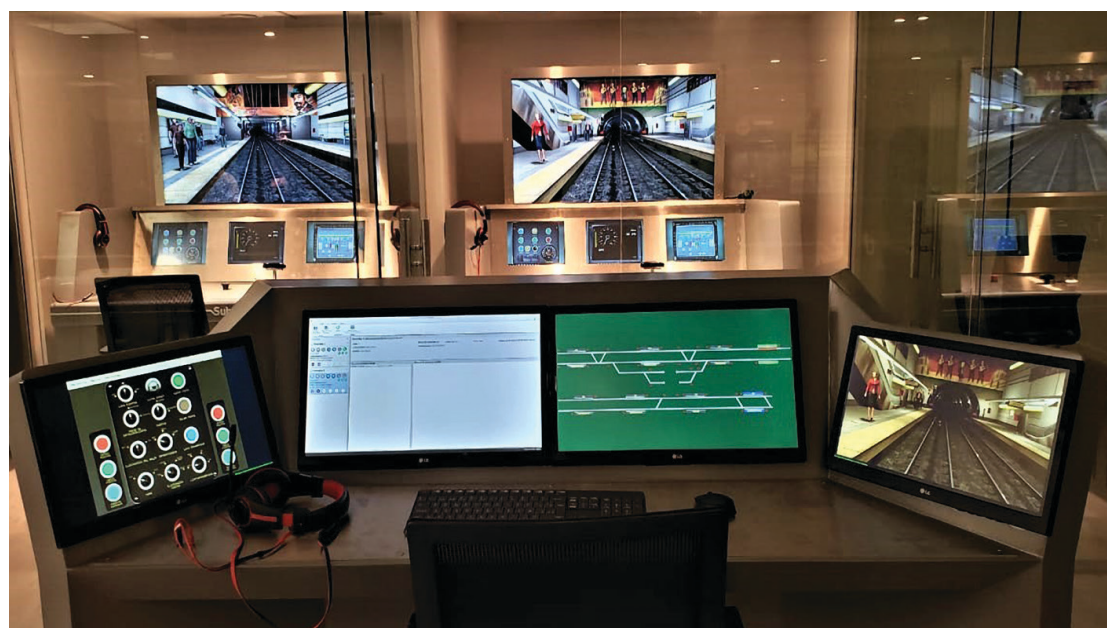

with invited professors from these and other internationally recognized research groups.

\section{The PLADEMA Institute}

The PLADEMA Institute ${ }^{c}$ depends on the School of Sciences of the Universidad Nacional del Centro de la Provincia de Buenos Aires (UNCPBA), the National Atomic Energy Commission (CNEA) and the Scientific Research Commission (CIC) of the Buenos Aires Province. It began its activities in 1997, standing out for providing solutions to applied problems using a multidisciplinary approach, providing its main contributions in the areas of simulation, mathematical modeling, high-performance computing, and graphics computing. These fields apply cross-cutting methodological tools to tackle different problems using complementary approaches, fostering the development of novel solutions of great impact from the social and productive point of view at the local, regional, and national levels. Currently, PLADEMA has six research teams focused on specific topics. The whole team consists of more than 100 members (20 Ph.D.'s, 18 doctoral fellows, four support personnel, and more than 60 interns). Three groups are actively working in image processing or computer graphics: Yatiris, researching problems related to medicine, including the processing of medical images; MediaLab, which specializes in computer graphics, virtual reality, and the development of training simulators; and RedDot, which focuses on video analysis, computer vision, and signal processing. The main researchers of these groups are Marcelo Vénere, Alejandro Clausse, Ignacio Larrabide, Cristian García-Bauza, and Juan D'Amato.

Some results from the Yatiris team in image processing include: automatic segmentation in magnetic resonance imaging (MRI) and computed tomography (CT) images for the detection and discretization of arteries and intravascular ultrasound images in order to perform hemodynamic modeling, and the use of deep learning techniques to detect retino-

c www.pladema.net 
blastomas in ocular images. ${ }^{16}$ Interesting advances were achieved in training areas with the implementation of an abdominal ultrasound simulator ${ }^{17}$ and the construction of anatomical tables for the teaching of anatomy.

The MediaLab team is a mainstay within Argentina when it comes to work in computer graphics and virtual reality. Innovative techniques to achieve highly realistic modeling effects have been developed by the group for use in real-time applications and video games; ${ }^{18}$ also, a computing platform that allows the real-time management of multiple projection surfaces was built, based on which the first Cave Automatic Virtual Environments (CAVEs) were developed in Argentina; MediaLab's multidisciplinary team achieved the successful CAVE implementation using Virtual Reality techniques. Interactive applications using video game concepts applied to the social and cognitive development of children with intellectual disabilities also have been used at the lab to allow learning through play. ${ }^{19}$

Finally, the RedDot team specializes in real-time image processing applied to computer vision and security applications. Through the use of deep learning techniques, the team has developed algorithms able to monitor scenes and detect the behavior of certain objects. ${ }^{20}$ Solutions have been implemented for urban traffic, movement of people, and detection of equipment failures in industrial plants.

PLADEMA is a strong actor in the transfer and development of applied technological products. Since 2001, PLADEMA has implemented more than 30 innovative products for Ministries, other government entities, and private companies, winning many national innovation awards. A good example of this kind of applied project is the development of a simulator covering the entire subway network of the city of Buenos Aires to train the drivers of subway trains how to handle system failures (Figure 3). Training with the simulator enables drivers to practice solving various types of faults on a varied set of trains, within a simulated virtual environment that emulates real processes. The project faced great technological challenges at the national and international levels, including the accurate modeling of the physical behavior of the trains, simulation of the movement of large crowds, representation of many kilometers of tunnels and tracks, and of the geometry of more than 100 railway stations. Furthermore, everything must work in real time.

The implementation of the RUBIKA platform is another success story that includes the construction of nine CAVEs, installed at different institutions in our country, along with the development of software packages for training in several areas, such as teaching in petroleum engineering careers, and the design of nuclear power plants (Figure 4). The goal was to design, build, and fully develop a local solution for several applications with the same quality as its international equivalents, taking advantage of working with local knowledge and lower costs. This required solving complex technical challenges concerning the hardware and software that make up the environment using low-cost projectors so the device can be easily maintained, and required the development of a software platform with substantial added value in terms of calibration, distributed processing, and optical corrections. The structure has to allow the adjustment of the four surfaces (three walls and the floor) and the calibration of the projectors to ensure that the projected images are less than one pixel in error. Each surface is handled by a separate computer, and all four processes must run synchronously. Finally, the position of the user's head must be detected in real time to generate the correctly corresponding projection.

PLADEMA keeps close collaborative relationships with internationally well-known groups and with several R\&D institutions in Argentina. Also, the institute regularly holds postgraduate classes given by professors from these and other outstanding research groups. PLADEMA is also a benchmark for local and regional training offers, especially regarding the implementation of academic proposals such as technical and short careers, which are planned and designed together with government and business stakeholders, such as the Municipality of Tandil City and the Chamber of Companies of the Computer Pole of Tandil (CEPIT).

\section{The Image Processing Laboratory}

The Image Processing Laboratory (PILab) is part of the Institute of Scientific and Technological Research in Electronics (ICYTE) ${ }^{\mathrm{d}}$ of the Universidad Nacional de Mar del Plata (UNMdP) and CONICET. It started in 1987 as part of the Image Analysis and

\section{d https://icyte.conicet.gov.ar}

Figure 4. RUBIKA is a software and hardware platform that generates immersive virtual environments for training.

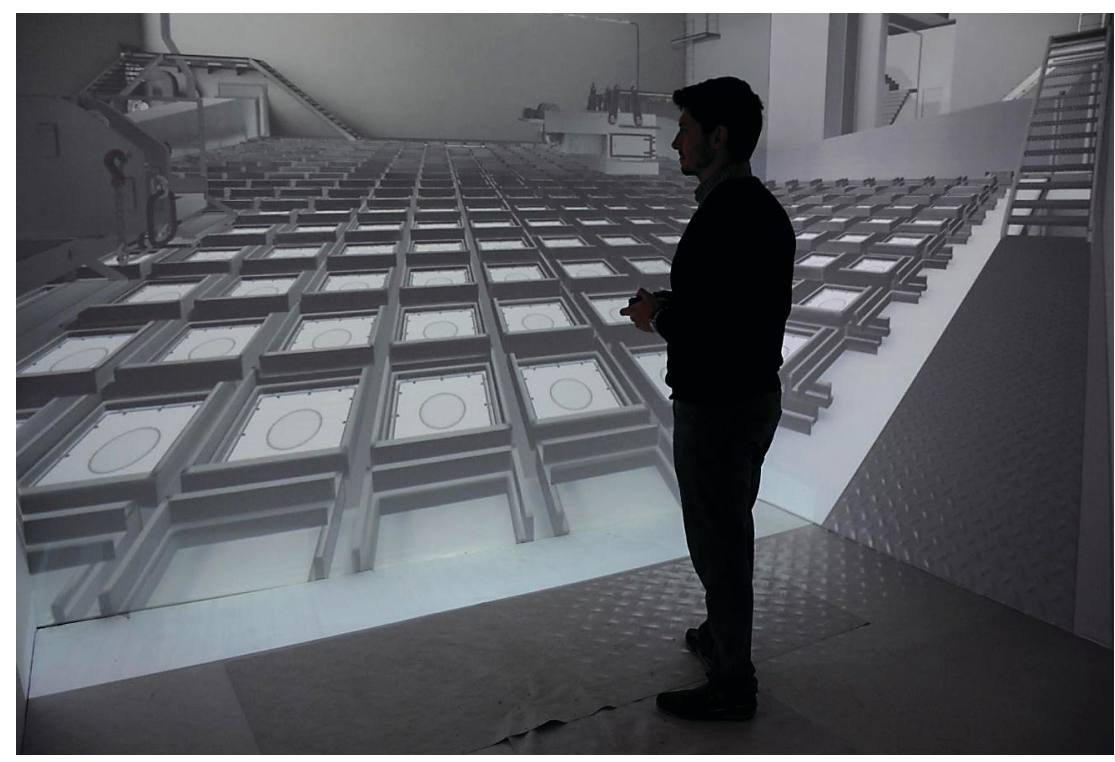


Figure 5. W-operators of the mathematical color morphology using diffuse definitions of color spaces: example of erosion and dilation applied to a color image. (a) Original color image. (b) Situation of the representative crisp colors in the RGB color space. (c) Volumes of colors in the 0.5-cut for the fuzzy colors yellow, blue, green, and gray obtained from the Voronoi diagram in the RGB cube. (d) Dilation. (e) Erosion.

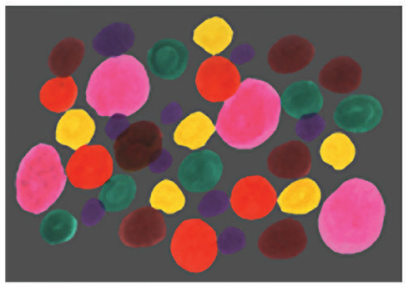

(a)

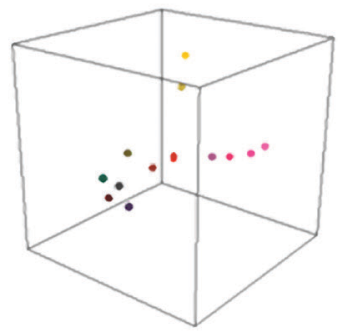

(b)

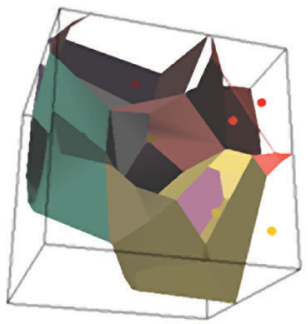

(c)

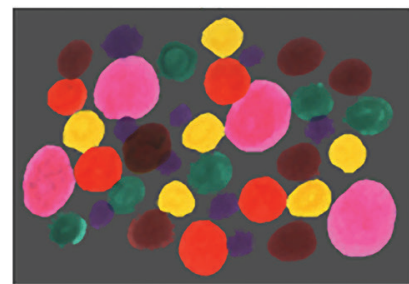

(d)

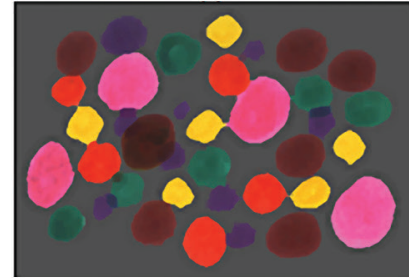

(e)
Coding Group (COANIM) of UNMdP, which later was dissolved since most members moved to the private sector. The remaining members created the Image Processing Group in the early 1990s. This group was strengthened with the addition of CONICET and CIC fellows, later formally becoming the PILab.

Since its creation, PILab has been nourished by numerous scholarships of all kinds, undergraduate students, CONICET and CIC Doctoral Scholarships, always maintaining a high number of junior participants (currently 50\%). More than half of the scholarship holders come from abroad, mainly from Ecuador and Colombia. Argentine scholarship holders typically decide to join as CONICET fellows, thus staying at the lab. The PILab currently has 20 members: eight seniors (Virginia Ballarin, Juan Pastore, Guillermo Abras, Eduardo Blotta, Agustina Bouchet, Marcel Brun, Diego Comas, and Inti Pagnucco), two postdocs, six Ph.D. candidates, and four interns. Its initial area of expertise was mathematical morphology, pioneering this topic in Argentina. Over time, the lab's goals shifted to the development of applications in medical imaging. Currently, three main research approaches coexist at the lab:

\section{Design of color morphological} operators and fuzzy morphological operators. Color is a very important visual feature in computer vision and image processing. The extension of grayscale image algorithms to color is not always direct. ${ }^{9,10}$ The PILab is developing theoretical advances in this direction, defining color morphological operators in a novel fuzzy color space (Figure 5). ${ }^{11,12}$ The automatic design of w-operators in gray levels and in color space is not only a theoretical approach of the lab, but these techniques also are being used to develop medical applications. ${ }^{13}$

\section{Deep learning models for seg-} mentation and knowledge discovery in medical images. From previous experience in discovering knowledge from data clustering using fuzzy systems, ${ }^{14}$ the new challenge regarding this paradigm is to be able to answer important questions about networks based on deep learning. Many of these questions have not been analyzed in the research literature. This research will allow us to study in depth the type of knowledge that can be extracted from answering questions such as:

- Is it possible to use the data representation contained in the internal parameters of the networks to extract interpretable knowledge?

- What is the meaning of these parameters, and how can we take advantage of them to extract knowledge?

- What does the internal data of the network describe regarding the problem addressed?

- How are changes in the input data reflected in the internal parameters of the networks?

\section{Tracking moving objects in} image sequences. Object tracking systems in image sequences have been

\section{Figure 6. Sperm motility tracker software.}

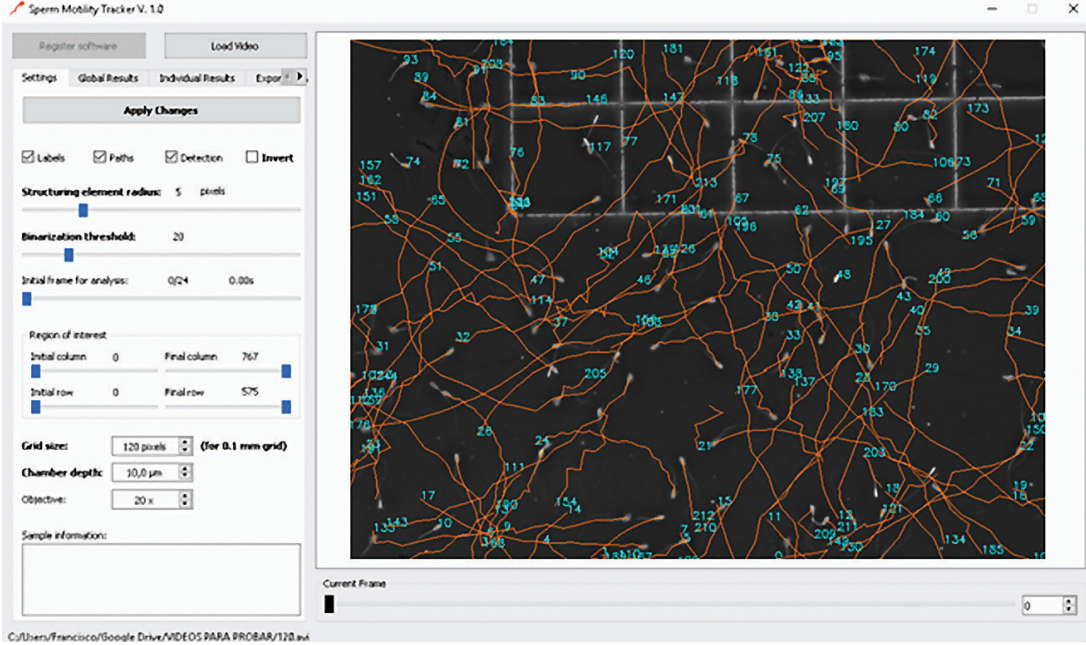


restricted to rigid objects. The image sequences from biological experimentation do not fit these models, which is why the PILab has increased its research efforts in tracking objects with other modes of movement, developing efficient methods to segment and quantify these objects. ${ }^{15}$

Apart from these research activities, the PILab has participated in various technological transfer initiatives ranging from fingerprints restoration for the Argentine Forensic Anthropology Team (EAAF), and DNA Images for Human Identification software for the Mar del Plata Human Genetics Foundation, to Sperm Motility Tracker software (Figure 6) for the Biological Research Institute (IIB).

The group maintains close collaboration with internationally renowned groups, including the College of Engineering of Texas A\&M University, U.S.; the Image processing Lab of the Instituto de Matemática e Estatística of the Universidade de São Paulo, Brazil; the CEATIC Center for Advanced Studies in Communication Technologies of the Universidad de Jaen, Spain, and the Uncertainty and Imprecision Modelling in Decision Making group of the Universidad de Oviedo, Spain. The PILab also maintains close collaboration with local centers of excellence to which the PILab transfers technology, such as the Institute of Biological Research (IIB-CONICET), the Plant Physiology Laboratory of the INTA Balcarce, and especially with the Institute of Research in Technology and Materials Science (INTEMA).

\section{Joint Activities and Conclusion}

Aiming to gain visibility as a discipline, these three groups jointly sponsor other activities which include the organization of a yearly School and Workshop in Imaging Sciences (ECIMAG), ${ }^{\mathrm{e}}$ and a joint Ph.D. program in medical imaging. The ECIMAG was held from 2008 until 2014, after which the financial situation of the scientific communities in Argentina suffered a huge shortage. During these years, the event was organized by universities in some of the major cities of Argentina, including Buenos Aires, Tandil, Bahía

e www.ecimag.org
Blanca, and Santa Fe. Apart from regular paper and poster presentations, the school would bring between two and four renowned invited professors from international research centers to deliver intensive courses focused on advanced topics related to image processing.

During these events, it became apparent that medical imaging was an important common research topic of the three research groups, while all three faced similar difficulties in developing Ph.D. programs in the area, due to the lack of critical mass in faculty. For this reason, a project for developing a joint Ph.D. program in the topic was presented and finally approved. In late 2018, the degree was opened at the Universidad Nacional del Sur, obtaining official accreditation from the corresponding Ministry Agency (CONEAU). Currently there are three candidates in the program at that university, with other candidates submitting applications. During 2019, the degree was opened at the Universidad Nacional de Mar del Plata and the Universidad Nacional del Centro de la Provincia de Buenos Aires, and their respective accreditations are due in the months to come.

While the field of imaging sciences started as a fringe area in engineering departments, it has matured and developed into a discipline in its own right in Argentina, as evidenced by the accomplishments of the three research groups. In spite of financial limitations, the groups have grown in research, development, and extended collaborations internationally, making the future of imaging science in Argentina look bright indeed.

\section{References}

1. Thomsen, F.S.L., Delrieux, C.A., and de Luis-García, R. Local texture descriptors for the assessment of differences in diffusion magnetic resonance imaging of the brain. Intern. J. Computer-Assisted Radiology and Surgery 12, 3 (2018), 389-398.

2. Navarro, P., et al. Body shape: Implications in the study of obesity and related traits. American J. Human Biology 32, 2 (2020).

3. Cintas, C., Quinto-Sánchez, M., Acuña, V., Paschetta, C., de Azevedo, S., and Delrieux, C. Automatic ear detection and feature extraction using geometric morphometrics and convolutional neural networks. IET Biometrics 6. 3 (2017), 211-223.

4. Avila, F., Delrieux, C., and Gasaneo, G. Complexity analysis of eye-tracking trajectories. The European Physical J. 92, 12 (2019)

5. Revollo, N.V., Delrieux, C.A., and González-José, R Set of bilateral and radial symmetry shape descriptor based on contour information. IET Computer Vision 11, 3 (2018), 226-236.

6. Sarmiento, G.N.R., Cipolletti, M.P., Perillo, M.M.,
Delrieux, C.A., and Perillo, G.M.E. Methodology for classification of geographical features with remote sensing images: Application to tidal flats. Geomorphology 257 (2018), 10-22.

7. Cipolletti, M.P., Genchi, S.A., Delrieux, C., and Perillo G.M.E. An approach for estimating border length in marine coasts from MODIS data. IEEE Geoscience and Remote Sensing Letters 17, 1 (2020), 8-12.

8. Genchi, S.A., Vitale, A.J., Perillo, G.M.E., Seitz, C., and Delrieux, C.A. Mapping topobathymetry in a shallow tidal environment using low-cost technology. Remote Sensing 12, 9 (2020).

9. Bouchet, A., Alonso, P., Pastore, J.I., Montes, S., and Díaz, I. Fuzzy Mathematical Morphology for color images defined by fuzzy preference relations. Pattern Recognition. Elsevier (Dec. 2016), 720-733, DOI: 10.1016/j.patcog.2016.06.014.

10. Pastore, J, Bouchet, J.A., Brun, M., and Ballarin, V. New windows-based color morphological operators for biomedical image processing. J. Physics. 705, 1 (Apr. 2016)

11. Bouchet, A., Colabella, L., Omar, S., Ballarre, J., and Pastore, J. Processing of microCT implantbone systems images using fuzzy mathematical morphology, J. Physics 705, (Apr. 2016).

12. Bouchet, A., Pastore, J.I., Marcel Brun, M., and Ballarin, V.L. Compensatory fuzzy mathematical morphology. Signal, Image and Video Processing 11, 6 (Sept. 2017), 1065-1072. Springer.

13. Pastore, J.I., Brun, M., Bouchet, A., and Ballarin, V.L. Color morphological reconstruction as a tool for microscope cell images. IFMBE Proceedings 60 (2017), 312-315. Springer International Publishing.

14. Comas, D.S., Pastore, J.I., Bouchet, A., Ballarin, V.L., and Meschino, G.J. Interpretable interval type-2 fuzzy predicates for data clustering: A new automatic generation method based on self-organizing maps. Knowledge-Based Systems 133, 1 (Oct. 2017), 234-254. Elsevier.

15. Imbachí, F.B. et al. Objective evaluation of ram and buck sperm motility by using novel sperm tracker software. Reproduction J. (May 22, 2018); DOI 10.1530/REP-17-0755.

16. Orlando, I., Van Keer, K., Breda, J.B., Manterola, H., Blaschko M., and Clausse, A. Proliferative diabetic retinopathy characterization based on fractal features: Evaluation on a publicly available data set. Amer Assoc Physicists Medicine Amerinst Physics 44, 12 (2017), 6425-6434.

17. Vitale, S., Orlando, I., Iarussi, E., and Larrabide, I Improving realism in patient-specific abdominal ultrasound simulation using CycleGANs. Intern. J. Computer-Assisted Radiology and Ssurgery, 2019, Springer.

18. D'Amato, J.P., García Bauza, C., Lazo, M., and V. Cifuentes, V. Tridimensional Scenes Management and Optimization for Virtual Reality simulators. Advances in Intelligent Systems and Computing 444 (2016), 243-252. Springer-Verlag, Heidelberg.

19. Contreras, M., García Bauza, C., and Santos, G. Videogame-based tool for learning in the motor, cognitive and socio-emotional domains for children with intellectual disability. Entertainment Computing 30 (2019), DOI: 10.1016/j.entcom.2019.100301.

20. Domínguez, L., D’Amato, J.P., Pérez, A., Rubiales, A and Barbuzza, R. A GPU-accelerated LPR algorithm on broad vision surveillance camera. J. Information Systems Engineering \& Management 3 (2018), 1-7.

Claudio Delrieux is a professor in the Departamento de Ing. Eléctrica y Computadoras, Universidad Nacional del Sur, Argentina, and Research Fellow in Consejo Nacional de Investigaciones Científicas y Tecnológicas/CONICET.

Virginia Ballarín is a professor with the ICYTE CONICET-CIC and on the Facultad de Ingeniería Universidad Nacional de Mar del Plata, Argentina.

Cristian García Bauza is a professor with PLADEMA CNEA - CICPBA - Facultad de Cs. Exactas, Universidad Nacional del Centro de la Prov. de Buenos Aires, Argentina, and Research Fellow in Consejo Nacional de Investigaciones Científicas y Tecnológicas/CONICET.

Mario A. López is Distinguished Professor at the Ritchie School of Engineering and Computer Science of the University of Denver, CO, USA.

(C) 2020 ACM $0001-0782 / 20 / 11$ 